\title{
A highly multiplexed biochemical assay for analytes in dried blood spots: application to newborn screening and diagnosis of lysosomal storage disorders and other inborn errors of metabolism
}

\author{
Xinying Hong, $\mathrm{BSC}^{1}$, Martin Sadilek, $\mathrm{PhD}^{1}$ and Michael H. Gelb, $\mathrm{PhD} \oplus^{1,2}$
}

Purpose: To develop a multiplexed assay for the newborn screening of lysosomal storage disorders and additional inborn errors in a flexible, comprehensive, and affordable manner to keep up with the expansion of the newborn screening panel.

Methods: Ultraperformance liquid chromatography-tandem mass spectrometry (UPLC-MS/MS) was chosen as the detection platform for its superiority compared to traditional flow-injection MS/MS.

Results: A high-throughput, 18-plex UPLC-MS/MS assay was developed for screening purposes with a sample turnaround time of 2.7 minutes. The assay was consolidated such that only four dried blood spot punches were required, and it displayed good precision and reproducibility.
Conclusion: We report a highly multiplexed UPLC-MS/MS assay that is appropriate for the newborn screening of 15 lysosomal storage diseases and 3 additional inborn errors. It can be further expanded to include additional conditions for which presymptomatic diagnosis may facilitate optimum treatment outcome.

Genetics in Medicine (2020) 22:1262-1268; https://doi.org/10.1038/s41436020-0790-9

Keywords: newborn screening; UPLC-MS/MS; multiplex; enzymatic assay; biomarker

\section{INTRODUCTION}

Every year, millions of newborns are screened for a panel of genetic disorders that are best treated if therapy is initiated prior to the onset of irreversible symptoms. Expansion of the newborn screening panel is inevitable due to the rapid development of innovative treatments for genetic diseases, and there has been widespread discussion of how to expand the panel in a flexible, comprehensive, and affordable manner. It is clear that the biochemical methodology will be the main way forward in the coming years, as genotyping-based screening cannot be currently adopted as a first-tier screen due to incomplete understanding of genotype/phenotype correlations, especially for rare diseases of which variant annotations are limited.

Tandem mass spectrometry (MS/MS) is by far the most comprehensive technique for biochemical analysis for analytes in dried blood spots (DBS), including enzymatic functions, ${ }^{1-5}$ biomarkers, ${ }^{6-9}$ and proteins of low abundance. ${ }^{10}$ Fluorometric assays, while useful for measuring some enzymatic activities in DBS, have limited applications in biomarker analysis. Immunoassays are useful for measuring abundant proteins, yet fall short of detecting proteins of extremely low concentration in DBS. They can also give rise to false-negative errors in the case of properly folded, but enzymatically inactive proteins. ${ }^{11}$

In newborn screening laboratories, the most common platform for MS/MS analysis is flow-injection MS/MS, where samples are introduced to the electrospray ionization (ESI) source directly by a continuous solvent flow without chromatography. Analytes are converted into ions in the heated ESI source and analyzed by MS/MS. However, flowinjection MS/MS analysis is inadequate to detect analytes of low abundance and to accurately quantify analytes when isobaric interferences are present. The alternative to flowinjection MS/MS is liquid chromatography (LC) MS/MS, where the in-line LC column provides additional analytical separation. LC-MS/MS also offers improved sensitivity flowinjection MS/MS as the column reduces ionization suppression effects and improves the signal-to-noise ratio for low concentration biomarkers.

Over the past decade, our laboratory has developed multiple MS/MS-compatible enzymatic substrates that are suitable for high-throughput screening. ${ }^{1-4,12,13}$ These substrates were strategically designed so that they are (1) highly enzyme specific, ${ }^{3,12,14}$ (2) highly sensitive as they ionize well in the ESI source and fragment through one major pathway in the gas

\footnotetext{
${ }^{1}$ Department of Chemistry, University of Washington, Seattle, WA, USA; ${ }^{2}$ Deparment of Biochemistry, University of Washington, Seattle, WA, USA. Correspondence:
} Michael H. Gelb (gelb@uw.edu) 
Table 1 Disorders and their relevant enzymes and biomarkers included in the 18-plex assay.

\begin{tabular}{ll} 
Disease & Protein defect \\
\hline MPS I & $\alpha$-L-lduronidase (IDUA) \\
\hline Pompe & Acid $\alpha$-glucosidase (GAA) \\
\hline Fabry & $\alpha$-Galactosidase A (GLA) \\
\hline Krabbe & Galactocerebrosidase (GALC) \\
Gaucher & $\beta$-Glucosidase (GBA) \\
\hline Niemann-Pick type A and B & Acid sphingomyelinase (ASM) \\
\hline Neuronal ceroid lipofuscinosis 2 (CLN2) & Tripeptidyl peptidase 1 (TPP1) \\
\hline MPS II & Iduronate-2-sulfatase (I2S) \\
\hline MPS IIIB & $\alpha$-N-Acetyl-glucosaminidase (NAGLU) \\
\hline MPS IVA & N-Acetylgalactosamine 6-sulfatase (GALNS) \\
\hline MPS VI & N-Acetylgalactosamine 4-sulfatase (ARSB) \\
\hline MPS VII & $\beta$-Glucuronidase (GUSB) \\
Neuronal ceroid lipofuscinosis 1 (CLN1) & Palmitoyl protein thioesterase I (PPT1) \\
\hline Wolman & Lysosomal acid lipase (LAL) \\
\hline Metachromatic leukodystrophy & Arylsulfatase A (ARSA) \\
\hline X-linked adrenoleukodystrophy & ABCD1 \\
\hline Classic galactosemia & Galactose-1-phosphate uridylyltransferase (GALT) \\
\hline Biotinidase deficiency & Biotinidase \\
\hline MPS mucopolysacchidosis. & \\
\hline
\end{tabular}

MPS mucopolysaccharidosis.

phase, ${ }^{15,16}$ and (3) highly multiplexable through MS/MS. In this study, we are also interested in biomarkers that were reported to be useful disease indicators, including C26:0-lysophosphotidylcholine (C26:0-LPC) for X-linked adrenoleukodystrophy (X-ALD) ${ }^{17}$ and C16:0-sulfatide for metachromatic leukodystrophy (MLD). ${ }^{8}$ Furthermore, lysosphingomyelin (lyso-SM) for Niemann-Pick A/B disease and lysoglobotriaosylceramide (lyso-Gb3) for Fabry disease may be useful secondary biomarkers to resolve the false-positive cases identified by the relevant enzymatic assays. ${ }^{18,19}$ These biomarkers accumulate in DBS when the relevant proteins are deficient.

Herein, we report a multiplex DBS assay for the detection of 18 genetic diseases using ultraperformance liquid chromatography MS/MS (UPLC-MS/MS). Treatments for these conditions are either available or are being developed and evaluated in ongoing clinical trials. We believe that LC-MS/MS is continuously expandable to include additional disorders and is the only platform that is capable of keeping up with the expected expansion of newborn screening panels brought by the rapid advancements in treatments.

\section{MATERIALS AND METHODS}

\section{Materials}

Whole blood from a healthy adult donor was collected in a $\mathrm{K}_{2}$ EDTA blood collection tube with consent. Lyso-SM, C26:0LPC, and lyso-Gb3 were purchased from Avanti Polar Lipids. Stock solutions of lyso-SM and lyso-Gb3 were prepared in methanol. Stock solution of C26:0-LPC was prepared in 2:1 (v:v) chloroform:methanol. Stock solutions of lyso-SM, C26:0LPC, and lyso-Gb3 were diluted 100-fold into the whole blood for a final concentration of $500 \mathrm{nM}, 500 \mathrm{nM}$, and $50 \mathrm{nM}$, respectively. For example, $10 \mu \mathrm{L}$ of $50 \mu \mathrm{M}$ lyso-SM, $50 \mu \mathrm{M}$ C26:0-LPC, and $5 \mu \mathrm{M}$ lyso-Gb3 each was added into $1 \mathrm{~mL}$ of blood. The spiked blood was inverted 10 to 20 times to distribute the biomarkers. DBS was prepared from the spiked blood and was used as a positive control. Quality control DBS (QC-Base pool, QC-Low, QQ-Med, and QC-High) for lysosomal storage disorders were acquired from the Centers for Disease Control and Prevention (CDC).

Isotope-labeled internal standards for lyso-SM and C26:0LPC were purchased from Avanti Polar Lipids. The synthesis of the remaining substrates and internal standards have been previously reported. . $^{2,12,13,16,20-22}$ The reagents for assaying IDUA (mucopolysaccharidosis [MPS] I), ${ }^{16}$ GAA (Pompe), ${ }^{20}$ GLA (Fabry), ${ }^{20}$ GALC (Krabbe), ${ }^{20}$ ASM (Niemann-Pick $\mathrm{A} / \mathrm{B}),{ }^{20}$ and GBA (Gaucher) ${ }^{20}$ were a generous gift from PerkinElmer (NeoLSD MSMS Kit). The reagents for assaying I2S (MPS II), ${ }^{16}$ NAGLU (MPS IIIB), ${ }^{2}$ GALNS (MPS IVA), ${ }^{2}$ ARSB (MPS VI), ${ }^{16}$ GUSB (MPS VII), ${ }^{2}$ PPT1 (CLN1), ${ }^{13}$ TPP1 (CLN2), ${ }^{2}$ LAL (Wolman), ${ }^{12}$ biotinidase (biotinidase deficiency), ${ }^{21}$ and GALT (classic galactosemia) ${ }^{21}$ were synthesized in our laboratory as described, as well as the isotope-labeled C16:0-sulfatide ${ }^{8}$ and lyso-Gb3. ${ }^{22}$ Additional sources of the reagents are listed in the Supplementary Information.

\section{Methods}

This study received University of Washington institutional review board (IRB) approval. A detailed assay protocol is provided in the Supplementary Information. In short, four DBS punches and seven incubations/extractions were required for the entire process. The first punch was used for the NeoLSD MSMS Kit (PerkinElmer). ${ }^{20}$ The second punch was for measuring biotinidase and GALT activity. ${ }^{21}$ The third 

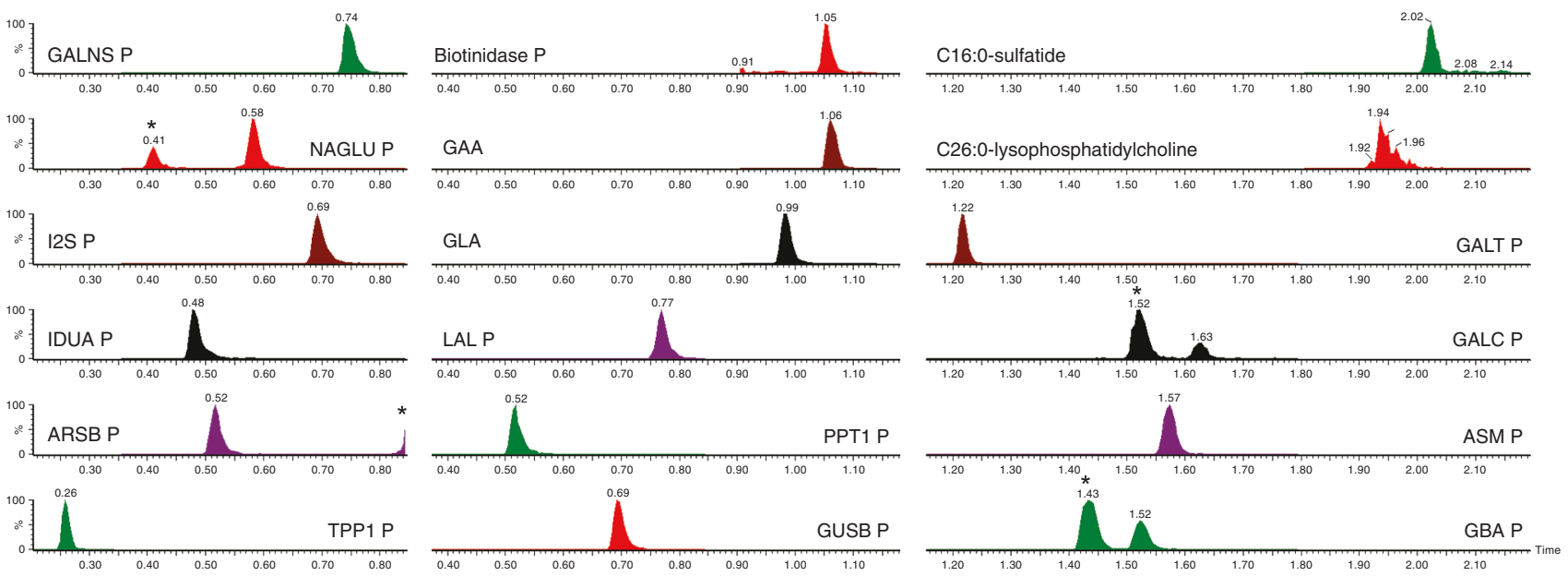

Fig. 1 Ultraperformance liquid chromatography-tandem mass spectrometry (UPLC-MS/MS) chromatogram of the 18-plex assay. The $x$-axis is time (minutes) and the $y$-axis is peak intensity after normalization to the highest peak in the channel (\%). The asterisks (*) indicate product peaks coming from in-source breakdown of substrates.

punch was extracted with water, and the blood extract was split three ways between assays for MPS II, IIIB, IVA, VI, VII, and CLN2; $;^{2}$ Wolman disease; ${ }^{12}$ and CLN $1 .{ }^{13}$ The fourth punch was for biomarker analysis and was extracted with methanol containing internal standards. The enzymatic assays were quenched and combined together, followed by a liquid-liquid extraction purification step. The purified sample was combined with the methanol extract before analyzing by multiple reaction monitoring (MRM) on a Xevo TQ mass spectrometer coupled to an Acquity UPLC system (Waters Corp.).

\section{RESULTS}

The 18 disorders targeted in this multiplex assay are listed in Table 1 together with their relevant enzymes and/or biomarkers. All conditions except X-ALD and MLD were tested by measuring the activity of the relevant enzyme. Classic galactosemia could be screened enzymatically in a dual test manner, where the first-tier test was the traditional quantitative Beutler assay (result ready on day 1), and the second-tier test was the novel GALT-LgtC coupled assay that are not affected by glucose-6-phosphate dehydrogenase (G6PD) (result ready on day 2). ${ }^{21}$ X-ALD and MLD were screened based on the abundance of the relevant biomarkers. For X-ALD, a functional assay of the relevant lipid transporter was not feasible. For MLD, the thermal instability of the relevant enzyme and the high frequency of pseudodeficiency variants posed additional challenges for enzymatic activity-based screening. ${ }^{23}$ Lyso-SM and lysoGb3 were also included in the assay and could serve as secondary disease indicators along with the enzymatic activity tests.

Combining multiple assays into the same reaction mixture minimizes the number of DBS punches needed as well as the pre-MS/MS steps. LAL and PPT1 are strongly inhibited by some blood components, therefore the assays for these enzymes required a DBS extraction step to alleviate the inhibition. $^{12,13}$ The biotinidase and GALT assay needed a separate punch as these two enzymes operate under nearneutral $\mathrm{pH}$ and are inactive at acidic $\mathrm{pH}$, which is the optimum for the lysosomal enzymes. ${ }^{21}$ Nonetheless, we envisioned that different assays could be combined together after their individual incubation/extraction and subjected to a single UPLC-MS/MS analysis to reduce instrumentation burden.

Since DBS on newborn screening cards are a limited resource, the assay was further consolidated to keep the number of DBS punches required to minimum. As only a small portion of the DBS extract is required for LAL and PPT1 assays, we hypothesized that it was possible to assay these two enzymes together with those for MPS II, IIIB, IVA, VI, VII, and CLN2 by splitting the blood extract. The enzymes from the DBS punch were extracted into water, and a small portion of the extract was transferred out for LAL and PPT1, while the rest of the extract and the punch was used for MPS II, IIIB, IVA, VI, VII, and CLN2. Although three separate incubations were performed, only one instead of three DBS punches was needed. Since the blood splitting approach diluted the substrate concentration for the multiplex assay for MPS II, IIIB, IVA, VI, VII, and CLN2, comparison studies between the whole punch method and the splitting method were carried to test its feasibility. As shown in Supplementary Fig. 1, TPP1, NAGLU, and ARSB activities were not affected by the blood extract splitting, indicating that the dilution of enzymatic substrates had little impact on their enzyme kinetics (saturating amounts of substrates are being used). Intriguingly, I2S, GALNS, and GUSB activities increased by $20-100 \%$ with the splitting method, which we speculated to be due to the dilution of some inhibitory blood components during the process. Together, the data indicated that LAL and PPT1 could be assayed together with MPS II, IIIB, IVA, VI, VII, and CLN2 using one DBS punch without compromising their performance. 

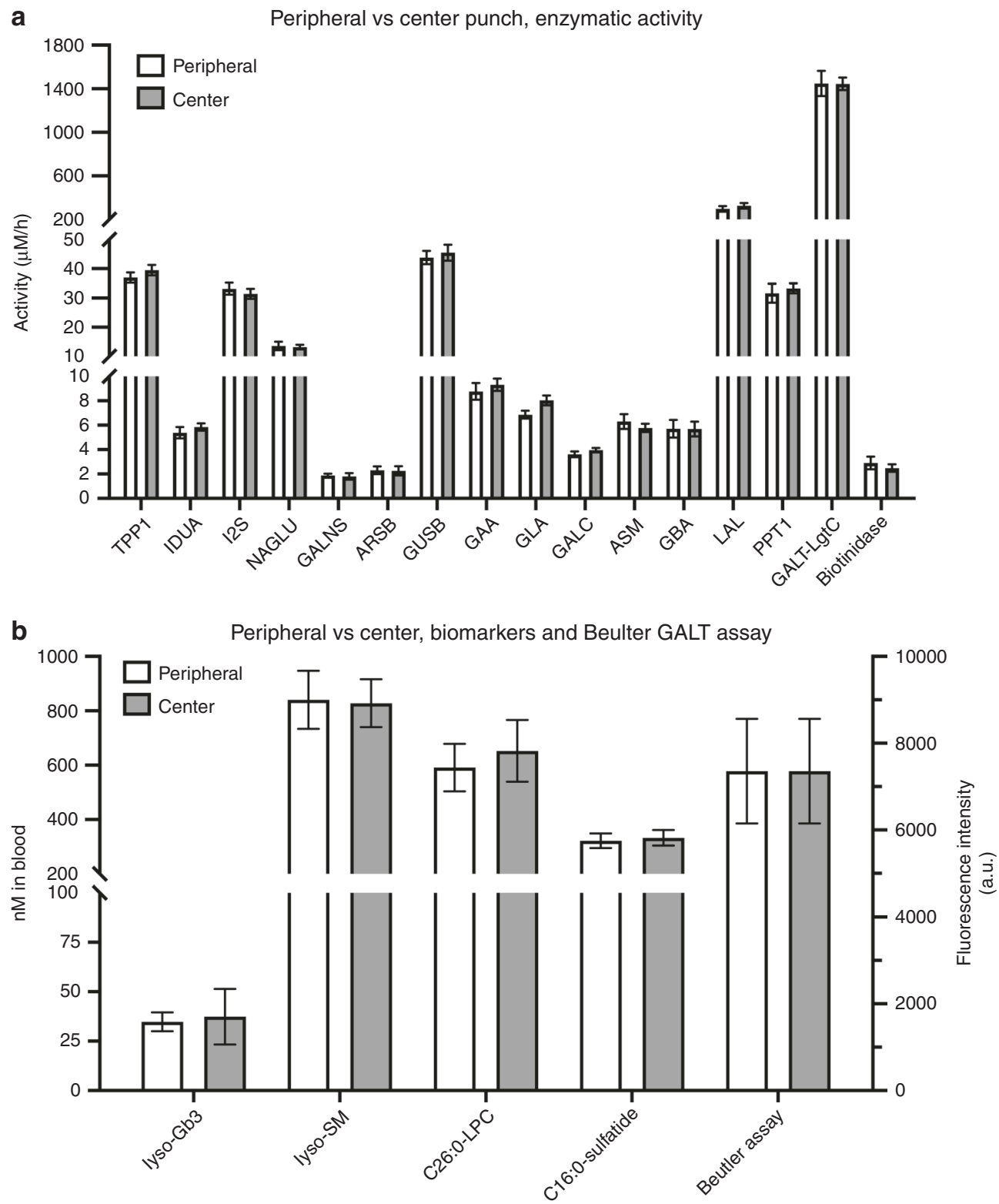

Fig. 2 Dried blood spot (DBS) sampling location study for the 18-plex assay. Results for (a) the enzymatic assays, and (b) the biomarker assays and the Beutler GALT assay. Ten replicates of peripheral punches and center punches were used in the study.

Shown in Fig. 1 is the UPLC-MS/MS chromatogram of the 18-plex assay. Each substrate and product pair as well as C26:0-LPC and its endogenous isobars were baseline resolved. The charged stationary phase of the column allowed separation between the sulfated substrate and the desulfated product of I2S, GALNS, and ARSB without ion-pairing reagents. Even though the heat-labile sulfated substrates underwent partial thermal breakdown in the heated ESI source and contributed significantly to their relevant product signal, it was of no concern as they were completely separated from their respective enzymatic products by chromatography. Column carryover and autosampler carryover were assessed and were negligible. The MRM channels were scheduled based on the retention time of each analyte to improve the duty cycle, though it was found that scanning 32 channels at the same time with a 5-millisecond dwell time and interchannel delay did not affect the signal significantly on our platform (data not shown). Columns from different batches were tested, with no significant difference found in the peak height, peak shape, and retention time for each analyte. Columns with high injection numbers displayed slightly higher backpressure (about $500 \mathrm{psi}$ ) and about 20\% loss in peak height when compared with a new column, with minimum shift in retention time. Nonetheless, chromatographic variations introduced by columns had negligible impact on the results, due to the use of chemically identical but isotopically distinguished internal standards. The sample injection-to-injection time of this 18-plex assay was 2.7 minutes, allowing more than 500 samples to be analyzed per day per instrument. 


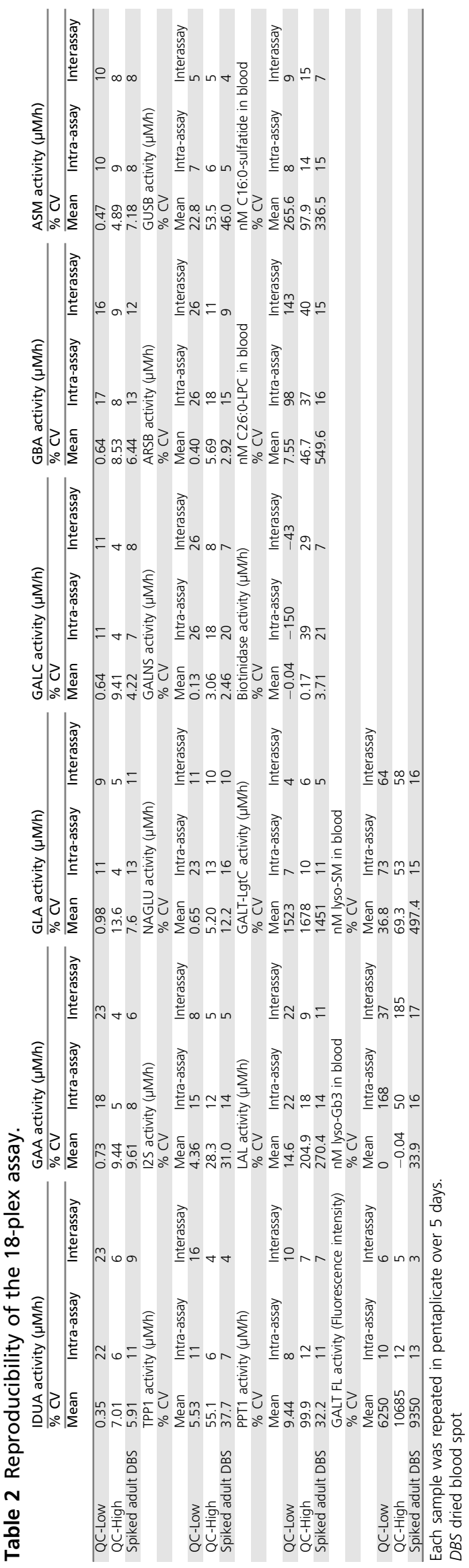

Kinetics and linearity studies as well as validations with patient samples were previously conducted for each assay, and were not repeated in the current study. ${ }^{1,2,12,13,20,21}$ There have been reports detailing the variations of DBS-based analysis introduced by hematocrit and sampling location. ${ }^{24,25}$ The bias introduced by sampling location was investigated by comparing the results obtained from ten center and ten peripheral punches from DBS prepared from whole blood spiked with biomarkers. As shown in Fig. 2, no statistically significant difference was found for all the analytes, suggesting that the chromatographic effect of the filter paper was negligible. The reproducibility and precision of the assay was assessed by analyzing QC-Low, QC-High, and spiked adult DBS in pentaplicates over 5 days (Table 2). All 14 lysosomal enzymes displayed good interday and intraday reproducibility at both the low and high end. The QC DBS from CDC did not constitute a positive control for the nonlysosomal enzymes (GALT and biotinidase) and the biomarkers except for C16:0sulfatide, thus spiked adult DBS was included as positive control for these disorders. Good reproducibility was found for the GALT and biotinidase activity as well as for the biomarkers with the spiked adult DBS (Table 2).

\section{DISCUSSION}

Flow-injection MS/MS was used as the detection platform for our early assays for lysosomal storage disorders. ${ }^{1}$ Flowinjection MS/MS works well for enzymatic assays for which the substrates undergo minimal thermal breakdown to enzymatic products in the ESI source. In-source conversion of substrates to products is not an issue with LC-MS/MS since the substrates can be chromatographically separated from enzymatic products, and thus this technique is more generally useful and most appropriate for highly multiplexed assays such as the 18-plex reported in this study. Flow-injection MS/ MS is often problematic for detection of low-abundant analytes in complex mixtures such as DBS due to the presence of one or more isobaric species. LC-MS/MS usually allows the analyte to be resolved on the column from isobaric interferences. With flow-injection MS/MS, all of the material extracted from DBS passes into the ESI source, resulting in the need for routine ESI source cleaning. This is a particularly important issue with newborn screening given the large volume of samples being analyzed. A key advantage of LCMS/MS is that the vast majority of the mass of material in DBS elutes from the LC column in the void volume and can thus be diverted away from the ESI source with a valve. LCMS/MS is more sensitive than flow-injection MS/MS since analytes suffer less ionization suppression due to matrix effects.

Recently, our laboratory and other groups have started to develop LC-MS/MS-based screening assays for newborn screening. ${ }^{2,26,27}$ The addition of LC to the MS/MS workflow does not significantly increase instrumentation complexity. Flow-injection MS/MS requires a single solvent delivery pump, whereas LC-MS/MS requires a second pump to allow for solvent gradients. An LC column is inserted into the same 
solvent line between the autosampler and the mass spectrometer as used in flow-injection MS/MS. All other components of the system are virtually identical, and the cost of the LC column is insignificant since one column can be used for several thousand newborns. LC-MS/MS-based assays are currently used in some newborn screening laboratories including Illinois, Washington, and Taiwan. ${ }^{28-30}$ It has been our experience in the Washington newborn screening laboratory that LC-MS/MS requires less routine cleaning of ESI source and internal mass spectrometer components (i.e., ion focusing lens, quadrupoles, and collision cells).

The assay reported here is highly multiplexed, with the ability to screen for 18 disorders simultaneously in a highthroughput manner. It is also highly robust as demonstrated by the reproducibility study and the consistency with columns from different batches and with different numbers of injections. Moreover, this 18-plex assay is continuously expandable for future addition of tests for new conditions, as the gradient covers a wide range of analytes with various hydrophobicity.

Most of our assays included a liquid-liquid extraction step to remove water soluble salts and detergents from the sample. However, it has been reported that LC-MS/MS-based enzymatic assays were able to work robustly in high volume laboratories without the liquid-liquid extraction as the salts and detergents were diverted to waste. ${ }^{29}$ Nevertheless, liquid-liquid extraction is still recommended in our multiplex assay as it can be hard to insert waste diversion windows when 18 analytes are eluting during most of the span of the run. In this study, 12-channel manual pipettes were used for all liquid transfers. Automation is possible for high volume laboratories, and therefore the assay can be carried out by one single laboratory worker.

Lyso-SM and lyso-Gb3 were included in our multiplexed panel as potential secondary disease indicators for Niemann-Pick A/B and Fabry disease, respectively. These markers were extracted together with the sulfatide and C26:0-LPC and were analyzed with the other analytes without adding additional complexity to the assay. Use of second-tier tests is often important for reducing the falsepositive rates of the primary tests, including psychosine for Krabbe disease, ${ }^{31}$ the ratio of creatine/creatinine for Pompe disease, ${ }^{32}$ and glycosaminoglycans for MPS I. Implementing lyso-Gb3 as a secondary screening filter may also reduce the false-negative rate of Fabry disease, a rare X-linked disorder caused by the deficiency of the GLA enzyme. Due to random $\mathrm{X}$-chromosomal inactivation, female carriers can have manifestation ranging from asymptomatic to as severely affected as males. ${ }^{33}$ It was reported that the GLA activity was normal or slightly decreased in at least $40 \%$ of the carriers. ${ }^{33}$ On the contrary, lyso-Gb3 was reported to be substantially elevated in symptomatic female carriers. ${ }^{33,34}$ Nevertheless, it remains to be proven if lyso-SM and lysoGb3 are indeed good biomarkers for Niemann-Pick A/B and Fabry disease, respectively. If so, by incorporating the additional biomarkers into the panel as secondary screening criteria, the screen-positive and screen-negative rates can be reduced.

Psychosine is an excellent biomarker for following up newborns with abnormally low GALC activities. ${ }^{35}$ However, it is not included in the current assay as our method cannot separate psychosine from its endogenous isobar, glucosylsphingosine (lyso-Gb1), which may also be a good biomarker for following up newborns with abnormal GBA activities. Moreover, psychosine analysis in DBS requires a top-end mass spectrometer, therefore a separate analysis may be more appropriate for this assay.

Amino acid, acylcarnitine, and succinylacetone analyses in DBS are crucial parts of newborn screening. Some laboratories derivatize these analytes prior to flow-injection MS/MS analysis. $^{36}$ These derivatives, however, cannot be analyzed on our multiplex platform as some of them are highly hydrophilic and elute in the void volume. We are currently working on new derivatization methods to be compatible with the LC-MS/MS method described in this study. By incorporating the amino acid, acylcarnitine, and succinylacetone analyses into our multiplex assay, instrumentation burden can be further reduced.

Probably the most significant hurdle to adopt LC-MS/MS in newborn screening laboratories is the sample turnaround time. An injection-to-injection time below 2 minutes is usually preferred, especially in laboratories with extremely high volume. Nonetheless, the increased turnaround time of the LC-MS/MS-based assay is offset by the consolidation of many individual assays into a single analysis, which are currently analyzed separately. We feel that consolidation is critical as newborn screening continues to expand due to the increasing rate of development of new treatments including gene therapies.

\section{SUPPLEMENTARY INFORMATION}

The online version of this article (https://doi.org/10.1038/s41436020-0790-9) contains supplementary material, which is available to authorized users.

\section{ACKNOWLEDGEMENTS}

This work was supported by the funding from the National Institutes of Health (R01 DK067859).

\section{DISCLOSURE}

M.H.G. is a cofounder of GelbChem, LLC, and a consultant for PerkinElmer. Award and filed patents include US20140249054A1, US20160298166A1, US8802833B2, EP2191006B1, and EP2385950B1. The other authors declare no conflicts of interest.

Publisher's note Springer Nature remains neutral with regard to jurisdictional claims in published maps and institutional affiliations. 


\section{REFERENCES}

1. Li Y, Scott CR, Chamoles NA, Ghavami A, Pinto BM, Turecek F, et al. Direct multiplex assay of lysosomal enzymes in dried blood spots for newborn screening. Clin Chem. 2004;50:1785-1796.

2. Liu $Y$, Yi F, Kumar $A B$, Chennamaneni NK, Hong $X$, Scott $C R$, et al. Multiplex tandem mass spectrometry enzymatic activity assay for newborn screening of the mucopolysaccharidoses and type 2 neuronal ceroid lipofuscinosis. Clin Chem. 2017;63:1118-1126.

3. Yi F, Hong $X$, Kumar AB, Zong C, Boons GJ, Scott CR, et al. Detection of mucopolysaccharidosis III-A (Sanfilippo syndrome-A) in dried blood spots (DBS) by tandem mass spectrometry. Mol Genet Metab. 2018;125:59-63.

4. Kumar AB, Hong X, Yi F, Wood T, Gelb MH. Tandem mass spectrometrybased multiplex assays for alpha-mannosidosis and fucosidosis. Mol Genet Metab. 2019;127:207-211.

5. Gelb MH, Lukacs Z, Ranieri E, Schielen P. Newborn screening for lysosomal storage disorders: methodologies for measurement of enzymatic activities in dried blood spots. Int J Neonatal Screen. 2019;5:1

6. Chace DH, Millington DS, Terada N, Kahler SG, Roe CR, Hofman LF. Rapid diagnosis of phenylketonuria by quantitative analysis for phenylalanine and tyrosine in neonatal blood spots by tandem mass spectrometry. Clin Chem. 1993;39:66-71.

7. Haynes CA, De Jesus VR. Improved analysis of C26:0lysophosphatidylcholine in dried-blood spots via negative ion mode HPLC-ESI-MS/MS for X-linked adrenoleukodystrophy newborn screening. Clin Chim Acta. 2012;413:1217-1221.

8. Spacil Z, Babu Kumar A, Liao HC, Auray-Blais C, Stark S, Suhr TR, et al. Sulfatide analysis by mass spectrometry for screening of metachromatic leukodystrophy in dried blood and urine samples. Clin Chem. 2016;62:279-286

9. Chace DH, Hillman SL, Van Hove JL, Naylor EW. Rapid diagnosis of MCAD deficiency: quantitative analysis of octanoylcarnitine and other acylcarnitines in newborn blood spots by tandem mass spectrometry. Clin Chem. 1997;43:2106-2113.

10. Jung S, Whiteaker JR, Zhao L, Yoo HW, Paulovich AG, Hahn SH. Quantification of ATP7B protein in dried blood spots by peptide immunoSRM as a potential screen for Wilson's disease. J Proteome Res. 2017; 16:862-871

11. Umapathysivam K, Whittle AM, Ranieri E, Bindloss C, Ravenscroft EM, van Diggelen OP, et al. Determination of acid $\alpha$-glucosidase protein: evaluation as a screening marker for Pompe disease and other lysosomal storage disorders. Clin Chem. 2000;46:1318-1325.

12. Masi S, Chennamaneni N, Turecek F, Scott CR, Gelb MH. Specific substrate for the assay of lysosomal acid lipase. Clin Chem. 2018;64:690-696

13. Khaledi H, Liu Y, Masi S, Gelb MH. Detection of infantile batten disease by tandem mass spectrometry assay of PPT1 enzyme activity in dried blood spots. Anal Chem. 2018:90:12168-12171.

14. Ghomashchi F, Barcenas M, Turecek F, Scott CR, Gelb MH. Reliable assay of acid sphingomyelinase deficiency with the mutation Q292K by tandem mass spectrometry. Clin Chem. 2015;61:771-772.

15. Spacil Z, Hui R, Gelb MH, Turecek F. Protonation sites and dissociation mechanisms of t-butylcarbamates in tandem mass spectrometric assays for newborn screening. J Mass Spectrom. 2011;46:1089-1098.

16. Chennamaneni NK, Kumar AB, Barcenas M, Spacil Z, Scott CR, Turecek F, et al. Improved reagents for newborn screening of mucopolysaccharidosis types I, II, and VI by tandem mass spectrometry. Anal Chem. 2014;86:4508-4514

17. Hubbard WC, Moser AB, Liu AC, Jones RO, Steinberg SJ, Lorey F, et al. Newborn screening for $X$-linked adrenoleukodystrophy (X-ALD): validation of a combined liquid chromatography-tandem mass spectrometric (LC-MS/ MS) method. Mol Genet Metab. 2009;97:212-220.
18. Chuang WL, Pacheco J, Cooper S, McGovern MM, Cox GF, Keutzer J et al. Lyso-sphingomyelin is elevated in dried blood spots of Niemann-Pick B patients. Mol Genet Metab. 2014;111:209-211.

19. Johnson $B$, Mascher $H$, Mascher $D$, Legnini E, Hung CY, Dajnoki $A$, et al. Analysis of lyso-globotriaosylsphingosine in dried blood spots. Ann Lab Med. 2013;33:274-278.

20. Elliott S, Buroker N, Cournoyer JJ, Potier AM, Trometer JD, Elbin C, et al. Pilot study of newborn screening for six lysosomal storage diseases using tandem mass spectrometry. Mol Genet Metab. 2016;118:304-309.

21. Hong $X$, Kumar $A B$, Ronald Scott $C$, Gelb MH. Multiplex tandem mass spectrometry assay for newborn screening of $X$-linked adrenoleukodystrophy, biotinidase deficiency, and galactosemia with flexibility to assay other enzyme assays and biomarkers. Mol Genet Metab. 2018;124:101-108.

22. Hong $\mathrm{X}, \mathrm{Gelb} \mathrm{MH}$. One-step synthesis of carbon-13-labeled globotriaosylsphingosine (lyso-Gb3), an internal standard for biomarker analysis of Fabry disease. Mol Genet Metab. 2018;125:292-294.

23. Tan MA, Dean CJ, Hopwood JJ, Meikle PJ. Diagnosis of metachromatic leukodystrophy by immune quantification of arylsulphatase $A$ protein and activity in dried blood spots. Clin Chem. 2008;54:1925-1927.

24. O'mara M, Hudson-Curtis B, Olson K, Yueh Y, Dunn J, Spooner N. The effect of hematocrit and punch location on assay bias during quantitative bioanalysis of dried blood spot samples. Bioanalysis. 2011;3:2335-2347.

25. Cozma C, lurascu MI, Eichler S, Hovakimyan M, Brandau O, Zielke S, et al. C26-Ceramide as highly sensitive biomarker for the diagnosis of Farber disease. Sci Rep. 2017;7:6149.

26. Spacil Z, Tatipaka H, Barcenas M, Scott CR, Turecek F, Gelb MH. Highthroughput assay of 9 lysosomal enzymes for newborn screening. Clin Chem. 2013;59:502-511.

27. Mechtler TP, Stary S, Metz TF, De Jesús VR, Greber-Platzer S, Pollak A et al. Neonatal screening for lysosomal storage disorders: feasibility and incidence from a nationwide study in Austria. Lancet. 2012;379:335-341.

28. Chan MJ, Liao HC, Gelb MH, Chuang CK, Liu MY, Chen HJ, et al. Taiwan national newborn screening program by tandem mass spectrometry for mucopolysaccharidoses types I, II, and VI. J Pediatr. 2019;205:176-182.

29. Burton BK, Charrow J, Hoganson GE, Waggoner D, Tinkle B, Braddock $S R$, et al. Newborn screening for lysosomal storage disorders in Illinois: the initial 15-month experience. J Pediatr. 2017:190:130-135.

30. Scott $C R$, Elliott $S$, Hong $X$, Huang $J Y$, Kumar $A B$, Yi F, et al. Newborn screening for mucopolysaccharidoses: results of a pilot study with 100 000 dried blood spots. J Pediatr. 2020;216:214-217.

31. Langan TJ, Orsini JJ, Jalal K, Barczykowski AL, Escolar ML, Poe MD. et al. Development of a newborn screening tool based on bivariate normal limits: using psychosine and galactocerebrosidase determination on dried blood spots to predict Krabbe disease. Genet Med. 2018;20:1644-1651.

32. Tortorelli S, Eckerman JS, Orsini JJ, Stevens C, Hart J, Hall PL, et al. Moonlighting newborn screening markers: the incidental discovery of a second-tier test for Pompe disease. Genet Med. 2018;20:840-846.

33. Nowak A, Mechtler TP, Desnick RJ, Kasper DC. Plasma LysoGb3: a useful biomarker for the diagnosis and treatment of Fabry disease heterozygotes. Mol Genet Metab. 2017;120:57-61.

34. Liao HC, Huang YH, Chen YJ, Kao SM, Lin HY, Huang CK, et al. Plasma globotriaosylsphingosine (lysoGb3) could be a biomarker for Fabry disease with a Chinese hotspot late-onset mutation (IVS4+919G>A). Clin Chim Acta. 2013;426:114-120.

35. Escolar ML, Kiely BT, Shawgo E, Hong X, Gelb MH, Orsini JJ, et al. Psychosine, a marker of Krabbe phenotype and treatment effect. Mol Genet Metab. 2017:121:271-278.

36. Turgeon C, Magera MJ, Allard P, Tortorelli S, Gavrilov D, Oglesbee D, et al. Combined newborn screening for succinylacetone, amino acids, and acylcarnitines in dried blood spots. Clin Chem. 2008;54:657-664. 